\title{
Chemo-hydro-geomechanics in acidizing assisted hydraulic fracturing
}

\author{
ManMan $\mathrm{Hu}^{1 *}$, Xiaojie Tang ${ }^{1}$ and Tomasz Hueckel ${ }^{2}$ \\ ${ }^{1}$ Deoartment of Civil Engineering, the University of Hong Kong, Hong Kong SAR \\ ${ }^{2}$ Department of Civil and Environmental Engineering, Duke University, Durham, USA
}

\begin{abstract}
Hydraulic fracturing has been widely used for unconventional reservoirs especially after the technique of horizontal drilling was invented. Acidizing treatment is often incorporated as a propagation enhancement of fractures, in particular, for very tight, low-permeability carbonate-rich reservoirs. How an individual crack propagates into a stressed medium subject to fluid pressure acting on the crack surfaces and meanwhile being affected by the chemically aggressive environment is still an open question. This short paper investigates the fundamental coupled chemo-hydro-geomechanics as encountered in typical scenarios of hydraulic fracturing, with a specific focus on the role of acidizing treatment. The constitutive relations consisting of a reactive-chemo-elastic and a reactive-chemo-plastic formulation are presented, followed by the coupling with geochemical processes, namely reactive-diffusion equations. Numerical investigations of two representative cases within the chemo-elastically defined regime are presented, featuring a laboratory injection test and in-situ stress conditions of a deep geothermal reservoir, respectively. The results have demonstrated that the chemical dissolution process plays a critical role in the distribution of circumferential stress around the crack tip as well as the evolution of crack propagation, and that acidizing treatment may accelerate cracking exponentially after sufficient chemical exposure.
\end{abstract}

\section{Introduction}

Although the technique of hydraulic fracturing has been developed for many years and has gained enormous success world-widely and particularly in USA, the modelling of this multiple-physics/chemical fracturing process falls far behind field applications. A common perspective is that the industry is simply manipulating the stimulation by trial and error. Poorly controlled acidizing or fracturing, often due to inadequate understanding of acid-water-rock interactions, has caused significant public concerns by not only posing threat and risks to the environment but also resulting in unnecessary costs and low productivity. This study investigates a fundamental problem, i.e. how the chemically aggressive environment affects crack propagation, in particular, how an individual crack propagates into a stressed geomaterial subject to a combined chemical and mechanical load.

By the definition in fracture mechanics, a crack extends when the stress intensity (indicated by the Jintegral) is no less than the material toughness. Hence subcritical crack propagation can be promoted by the following means: 1) enhancing stress localization in the vicinity of the crack tip, via e.g. decreasing the energy potential for generating new surfaces of micro-cracks in the process zone around the tip; or 2) lowering the material toughness or damage tolerance (via e.g. chemical degradation induced by mass removal) in front of the crack tip. Acidizing treatment was invented for the latter purpose, but the induced coupled physio-chemical processes were found playing an important role on the local stress redistribution around the crack tip, as well as the evolution of the stress intensity factor. In what follows, we outline the constitutive formulation of the chemo-hydro-geomechanics theory considering both the elastic and plastic domain of the rock behaviour and the governing law of the geochemical processes, and present case studies subsequently.

\section{Constitutive formulation}

The material subject to deformation and chemical mass exchange is modelled as elasto-visco-plastic, and the total strain rate is decomposed into elastic and plastic components:

$$
\dot{\varepsilon}_{i j}=\dot{\varepsilon}_{i j}^{e l}+\dot{\varepsilon}_{i j}^{p l}
$$

\footnotetext{
$\overline{\text { * Corresponding author: mmhu@hku.hk }}$
} 


\subsection{Chemo-elasticity}

The elastic strain rate $\dot{\varepsilon}_{i j}^{e l}$ is assumed to follow a chemoelasticity formulation proposed in [1], in analogy to thermo-elasticity:

$$
\dot{\varepsilon}_{i j}^{e l}=D_{i j k l}^{e} \dot{\sigma}_{i j}+\tilde{\alpha} \dot{\xi},
$$

where $D_{i j k l}^{e}$ denotes the elastic compliance tensor, $\dot{\xi}$ denoting the chemical dissolution rate. $\tilde{\alpha}$ denotes the chemical shrinkage coefficient, which is locally affected by the evolving microscopic structure and hence is described as a function of deformation $\tilde{\alpha}=\alpha_{0}(1+\eta \hat{\varepsilon})$, where $\alpha_{0}$ and $\eta$ are coefficients denoting background chemical shrinking and microfracturation enhancement on the shrinkage, respectively. $\hat{\varepsilon}$ denotes a strain invariant, serving as a proxy for local mechanical damage. It is emphasized here that the terminology "chemo-elasticity" is regardless of thermodynamic implications, but originates from the quasi-linear behavior within a limited range of stress.

\subsection{Chemo-plasticity}

The irreversible plastic strain rate is assumed to follow an associative visco-plastic flow law :

$$
\dot{\varepsilon}_{i j}^{p l}=\dot{\lambda} \frac{\partial f}{\partial \sigma_{i j}},
$$

where $f$ denotes the yield function and $\dot{\lambda}$ is a scalar multiplier representing the magnitude of plastic strain rate, expressed as

$$
\dot{\lambda}=\sqrt{\dot{\varepsilon}_{q}^{p l^{2}}+\dot{\varepsilon}_{v}^{p l^{2}}},
$$

where $\dot{\varepsilon}_{q}^{p l}$ and $\dot{\varepsilon}_{v}^{p l}$ are the deviatoric and volumetric invariants of the plastic strain rate, respectively, following the Perzyna's overstress model :

$$
\left\{\begin{array}{l}
\dot{\varepsilon}_{q}^{p l}=\dot{\varepsilon}_{0}\left\langle\frac{q-q_{Y}}{\sigma_{r e f}}\right\rangle^{m}, \\
\dot{\varepsilon}_{v}^{p l}=\dot{\varepsilon}_{0}\left\langle\frac{p \prime-p_{Y}}{\sigma_{\text {ref }}}\right\rangle^{m},
\end{array}\right.
$$

where $\dot{\varepsilon}_{0}$ denotes a reference strain rate, $q$ and $p^{\prime}$ denoting the equivalent deviatoric stress and the volumetric mean effective stress, respectively. $\langle\cdot\rangle$ denotes the Macaulay brackets. $q_{Y}$ and $p_{Y}$ denote the deviatoric and normal components of the yield stress, respectively, and are obviously both susceptible to the chemical process.

The effect of chemical mass removal on the yield surface [2] is assumed linear for simplicity:

$$
\left\{\begin{array}{l}
q_{Y}=q_{Y 0}\left(1-\beta_{q} \xi^{\text {REV }}\right), \\
p_{Y}=p_{Y 0}\left(1-\beta_{p} \xi^{\text {REV }}\right),
\end{array}\right.
$$

where $q_{Y 0}$ and $p_{Y 0}$ are the deviatoric and normal components, respectively, of the initial yield stress. $\beta_{q}$ and $\beta_{p}$ are constant coefficients for the effect of chemical weakening on the respective yield stress component. It is emphasized that here $\xi^{R E V}$ is an averaged variable, denoting the accumulated mass removal (i.e. a time integration of the local mineral dissolution rate) averaged within a representative elementary volume (REV), and hence

$$
\xi^{R E V}=\int_{0}^{t}(1+\eta \hat{\varepsilon}) \dot{\xi} d \tau .
$$

The intricate relationship between the mass exchange rate at different scales, e.g. $\dot{\xi}^{R E V}$ and $\dot{\xi}$, in the context of phase transition induced transient instability is further explored in [3].

\section{Effect of geochemical processes}

Here we consider the predominant chemical process during acidizing for carbonate reservoirs to be calcite dissolution. Solute transport is described by the reactive diffusion equation with a nonlinear source term:

$$
\partial_{t} x_{\mathrm{Ca} 2+}=D_{\mathrm{Ca} 2+} \nabla^{2} x_{\mathrm{Ca} 2+}+\dot{\xi},
$$

where $x_{\mathrm{Ca2}}$ denotes the molar fraction of calcium in the fluid phase within the process zone, $D_{\mathrm{Ca} 2+}$ denoting the calcium diffusion coefficient.

Based on the kinetics of calcite dissolution, the dissolution rate is dependent of the local hydrogen concentration. According to [4], the local chemical mass removal rate can be written as

$$
\dot{\xi}=\beta_{H+}\left(C_{H+}\right)^{k \prime} .
$$

where $\beta_{H+}$ denotes a constant representing the effect of local acidity on the mass dissolution rate. $k^{\prime}$ is an exponential index, which is usually assumed as a constant with the value between $0.5 \sim 1.0$ [4].

Meanwhile the chemical dissolution rate is coupled to the reactive diffusion of the local hydrogen concentration, described as

$$
\partial_{t} C_{H+}=D_{H+} \nabla^{2} C_{H+}-\gamma_{C H} \dot{\xi},
$$

where $C_{H+}$ denotes the local concentration of hydrogen, $D_{H+}$ denoting the hydrogen diffusion coefficient. $\gamma_{C H}$ is a proportionality constant of the mass exchange rate of hydrogen consumption over that of calcium production in the fluid phase.

\section{Results and discussion}

Two cases that simulate a typical laboratory condition and a geothermal field condition, respectively, are investigated, with key parameters obtained from the literature $[5,6,7]$. The chemo-elastically affected zone is the centre of this study and the deviatoric strain invariant is chosen to be a proxy for the local mechanical damage. Compression is defined as positive.

As this study focuses on the effect of mineral mass removal on the process zone mechanical behaviour, rather than the opposite, the chemical processes inside the selected zone, including the pathways for ion diffusion and solute transport, are assumed as axisymmetric around the singularity (i.e. the crack tip point) for simplicity. 
Chemical boundary conditions for both cases are specified as: the acid concentration is kept constant at the crack tip and vanishing at the exterior; the concentration of calcium ion at the remote exterior boundary is zero; the dissolution rate at the centre is assumed controlled by the fluid $\mathrm{pH}$.

In the first case, the mechanical boundary conditions applied on the chemo-elastically defined process zone are assumed as follows. The interior pressure is assumed with the value of $p_{a}=3 \mathrm{MPa}$, which simulates a typical injection pressure applied in laboratory conditions. The exterior and the crack surfaces are traction-free. As all the fields involved are symmetric with respect to the $\theta=0^{\circ}$ axis, only the $0^{\circ} \sim 180^{\circ}$ part of the results will be displayed in the following discussions.

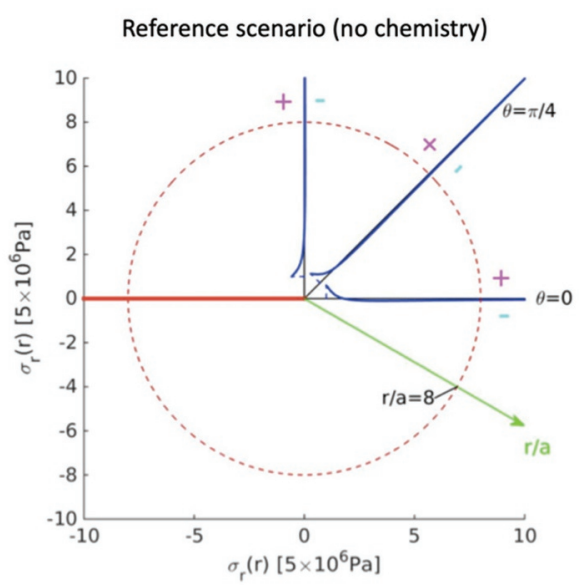

(a)

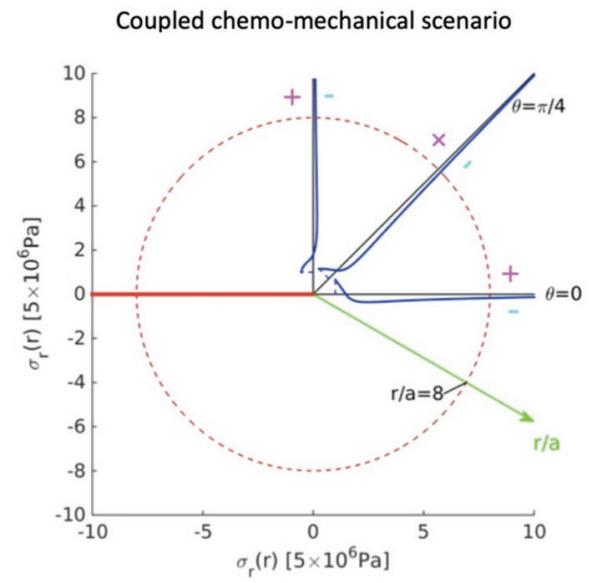

(b)

Fig. 1. Radial stress distribution along the radius at $\theta=$ $0^{\circ}, 45^{\circ}, 90^{\circ}$ around the crack tip: (a) a reference scenario with no chemistry involved and (b) a coupled chemo-mechanical scenario, for laboratory injection tests of carbonates.

Fig. 1 shows the radial stress distribution along the radius at $\theta=0^{\circ}, 45^{\circ}, 90^{\circ}$ around the crack tip point (with a small imaginary cavity assumed to eliminate the singularity). Fig. 1a provides a reference distribution when no chemistry is taken into account. Fig. 1b shows the counterpart after $700 \mathrm{~h}$ (around one month) chemical exposure. The effect of mineral dissolution on the radial stress distribution is noticeable but insignificant, due to the imposed boundary condition constraints. Note that Fig. 1a and Fig. 1b show a zoom-in of the process zone with the normalized radius $\in[1,8]$.

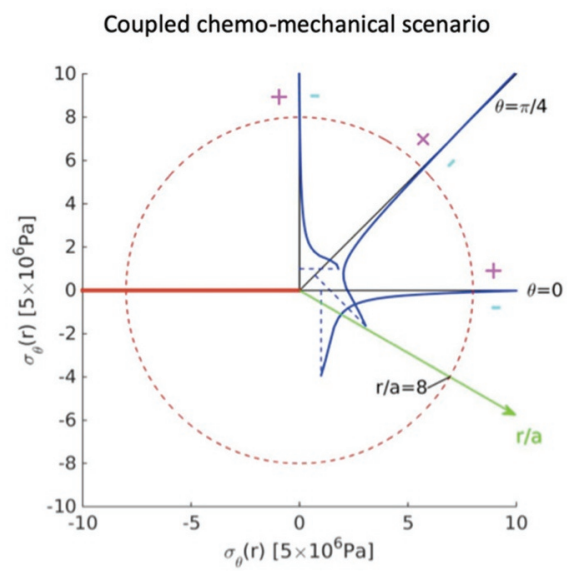

(a)

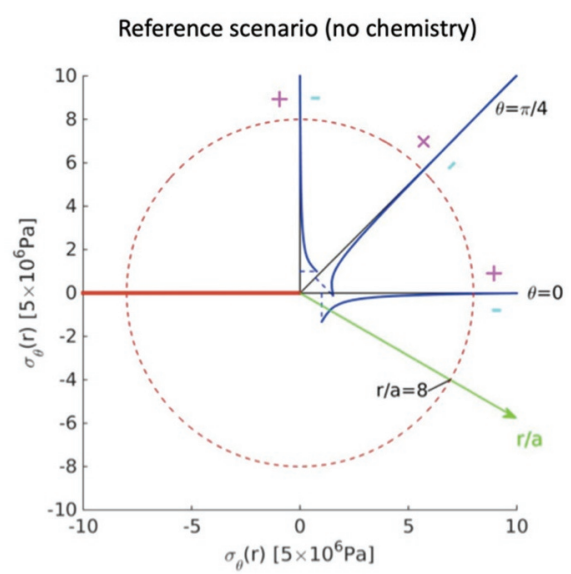

(b)

Fig. 2. Circumferential stress distribution along the radius at $\theta=$ $0^{\circ}, 45^{\circ}, 90^{\circ}$ around the crack tip: (a) considering chemomechanical coupling effect and (b) without any chemistry, for laboratory injection tests of carbonates.

However, that's not the case with the circumferential stress distribution in front of the crack tip, which is the most salient feature from the perspective of crack mechanics. Fig. 2 shows the comparison between the circumferential stress distribution along the radius at $\theta=$ $0^{\circ}, 45^{\circ}, 90^{\circ}$ in the process zone subject to a combined chemical and mechanical load for $700 \mathrm{~h}$ and the counterpart undergoing the mechanical load solely for the same duration.

Comparing Fig. 2a and Fig. 2b, we see that in both scenarios, the maximum tensile (indicated as negative) 
circumferential stress along the radius locates at the inner boundary and the absolute value decreases as $\theta$ increases (from $0^{\circ}$ to $90^{\circ}$ ) because the circumferential stress is forced to vanish on $\theta=180^{\circ}$. We report that with chemo-mechanical coupling (Fig. 2a), the maximum circumferential stress (in tension) at $\theta=0^{\circ}$ reads 18.6 $M P a$, which is significantly higher than that of the reference scenario without any chemistry (Fig. 1b), namely 6.9 MPa. Note also that there is a characteristic hump in the stress distribution near the inner boundary in the coupled scenario, corresponding to a localized distribution of the overall mass removal around the crack tip.

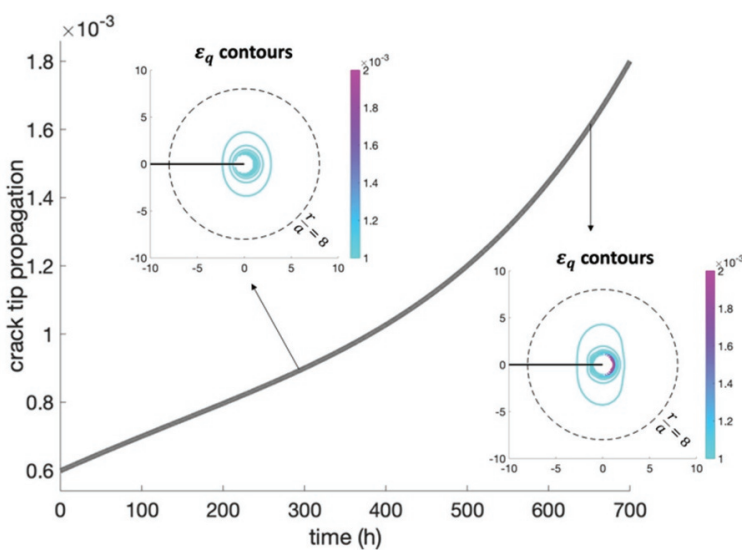

Fig. 3. Crack tip propagation over time with the contour patterns of the deviatoric strain invariant distribution juxtaposed. When the crack propagation starts to grow exponentially, the deviatoric strain (i.e. local damage) distribution becomes localized in front of the crack tip.

Fig. 3 shows the evolution of crack tip propagation with time with damage-dissolution coupling inside the process zone considered. A significant nonlinearity is observed, and the propagation accelerates exponentially after $500 \mathrm{~h}$ chemical exposure, which corresponds to an enhanced shear and a much more localized distribution of damage in front of the crack tip.

Now we move on to discuss the second case. The chemical boundary conditions and material parameters are kept the same, for the sake of comparison. The mechanical boundary conditions in this case are closer to the in-situ scenarios. The imposed stresses on the crack surfaces and the imaginary ring are assumed to be $p_{a}=$ $p_{f}=23 \mathrm{MPa}$. The exterior boundary condition is $p_{b}=$ $18 M P a$. These values are taken from the field data set of a geothermal reservoir at the depth of around $1 \mathrm{~km}$.

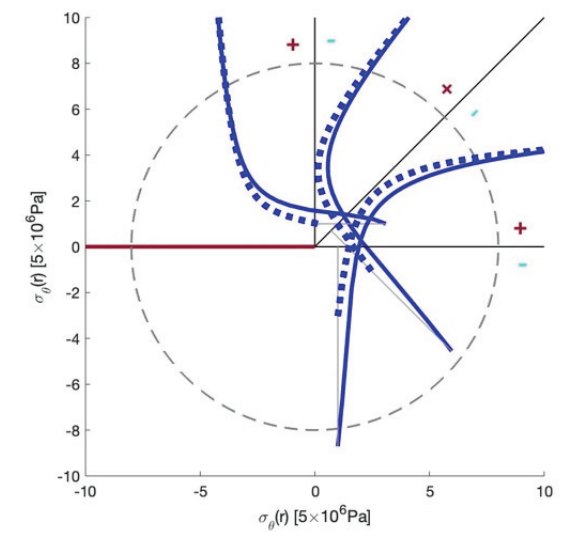

Fig. 4. Circumferential stress distribution along the radius at $\theta=$ $0^{\circ}, 45^{\circ}, 90^{\circ}$ around the crack tip: solid curves showing the stress distribution after one month of mineral dissolution while the dashed curves indicating the stress distribution without any dissolution, for in-situ stress conditions.

The circumferential stress distribution around the crack tip is demonstrated in Fig. 4, with the solid curves showing the stress distribution along the radius at $\theta=$ $0^{\circ}, 45^{\circ}, 90^{\circ}$ after one month of mineral dissolution while the dashed curves indicating the counterpart distribution without any dissolution as a reference. Compared to the results from the first case (see Fig. 2), the maximum tensile stress on the inner boundary increases dramatically as a result of the increase in imposed stress. A striking difference is that the circumferential stress in this case is not uniformly tensile anymore. In the tensile zone, the circumferential stress changes rapidly along the radius while it transits to a much smoother distribution in the compressive zone. As shown in Fig. 4, the characteristic hump in the stress distribution near the inner boundary is clearly widened by the chemical process.

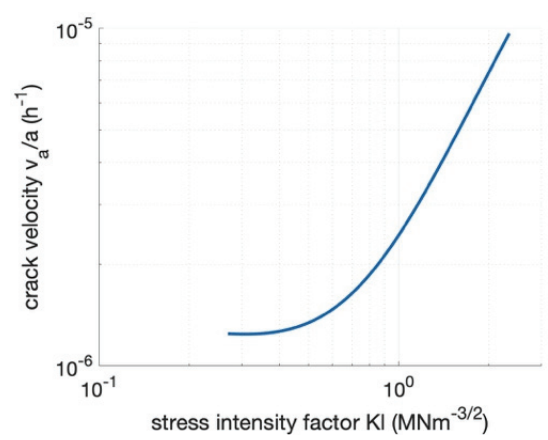

Fig. 5. Crack propagation rate versus mode I stress intensity factor in logarithmic scale considering chemo-mechanics.

Eventually, the crack propagation rate and the stress intensity factor (mode I) at the crack tip in an acidizing scenario are correlated and shown in Fig. 5 in logarithmic scale. The crack propagation rate is enhanced exponentially with the increase of the tensile stress intensity factor at the crack tip as a result of stress redistribution significantly affected by the chemical process. A hypothesis is that the contribution of chemical 
reaction process (here the diffusion process is suppressed) on driving the enhancement of crack propagation grows, by lowering the energy potential for generating the same amount of new surfaces of micro-cracks per unit volume around the tip.

\section{Conclusions}

In this short paper, we have outlined the theory of chemohydro-geomechanics for acidizing assisted hydraulic fracturing in terms of formulating the constitutive relations and the effect of geochemical processes (only mineral dissolution is considered), followed by numerical investigations of two cases within the chemo-elastically affected zone. The results have demonstrated that the chemical dissolution process plays a critical role in determining the evolution of crack propagation and stress redistribution, and that acidizing treatment can accelerate cracking in both cases. The effect of acidizing on the chemo-plasticity zone, as well as an evolution law for the elasto-plastic interface when subject to mineral mass removal, are currently under investigation. Future work will include the feedback mechanisms between coupled processes of chemical softening by mineral mass removal, irreversible damage manifested as micro-cracking, microchannelling induced alteration in local hydraulic conductivity and the delivery of acid, etc.

\section{References}

1. M.M. Hu and T. Hueckel. 2019. Modeling of subcritical cracking in acidized carbonate rocks via coupled chemo-elasticity. Geomechanics for Energy and the Environment. 19: 100114.

2. M.M. Hu and T. Hueckel. 2013. Environmentally Enhanced Crack Propagation in a Chemically Degrading Soil/Rock Mass. Géotechnique. 63(4): 313-321.

3. M.M. Hu, C. Schrank, K. Regenauer-Lieb. 2019. Cross-diffusion waves in hydro-poro-mechanics. Journal of the Mechanics and Physics of Solids. 135: 103632.

4. E.L. Sjöberg. 1976. A fundamental equation for calcite dissolution kinetics. Geochimica et Cosmochimica Acta. 40(4): 441-447.

5. M.O. Ciantia, R. Castellanza, G.B. Crosta, T. Hueckel. 2015. Effects of mineral suspension and dissolution on strength and compressibility of soft carbonate rocks. Engineering Geology. 184 : 1-18.

6. B. Lecampion and E. Detournay. 2007. An implicit algorithm for the propagation of a hydraulic fracture with a fluid lag. Computer Methods in Applied Mechanics and Engineering, 196(49-52): 4863-4880.

7. S. Kelkar et al. 2012. Modeling coupled thermalhydrological-mechanical processes during shear stimulation of an EGS well. In: Proceedings of the $37^{\text {th }}$ Workshop on Geothermal Reservoir Engineering, Stanford University. SGP-TR-194 